\title{
Thymic Adenocarcinoma with Positivity for Thyroid Transcription Factor-1 and a BRAF V600E Mutation: A Case Report
}

\author{
Takafumi Yorozuya ${ }^{1}$, Mitsuo Otsuka ${ }^{1}$, Shin Ichihara ${ }^{2}$, Kento Fujimori ${ }^{1}$, Chikako Kitamura ${ }^{1}$, \\ Tomoyuki Takahashi ${ }^{1}$, Masaki Mori ${ }^{1}$, Yasushi $\mathrm{Cho}^{3}$ and Hirofumi Chiba ${ }^{4}$
}

\begin{abstract}
:
Thymic adenocarcinomas are rare. We herein report for the first time a case of thymic adenocarcinoma with positivity for thyroid transcription factor-1 (TTF-1) and a BRAF V600E mutation. A 50-year-old woman had persistent suffocation and chest pain. Computed tomography revealed a $42 \times 28-\mathrm{mm}$ tumor in the anterior mediastinum. The patient underwent tumor resection using video-assisted thoracoscopic surgery. Histopathological findings showed thymic papillary adenocarcinoma, Masaoka stage II. Immunohistochemically, the tumor was positive for TTF-1. A sequencing analysis revealed a BRAF V600E mutation. The patient underwent postoperative radiotherapy and was in good health without recurrence at five months after resection.
\end{abstract}

Key words: thymus, thymic carcinoma, thymic adenocarcinoma, BRAF V600E, thyroid transcription factor-1

(Intern Med Advance Publication)

(DOI: 10.2169/internalmedicine.7623-21)

\section{Introduction}

Thymic carcinoma is a thymic epithelial tumor with cellular atypia (1) and accounts for approximately $9-18 \%$ of all thymic epithelial tumors (2-4). The most common histological type of thymic carcinoma is squamous cell carcinoma, and adenocarcinomas of thymic origin are rare, reported in only $3-4 \%$ of all thymic carcinomas $(5,6)$. The immunohistochemical expression of thyroid transcription factor-1 (TTF1) is a specific marker of lung and thyroid carcinomas. Previous reports have shown that almost all thymic adenocarcinomas have a negative TTF-1 expression, with only one report showing a positive expression (7).

BRAF V600E is the most common driver mutation in malignant melanoma and occurs in almost all cases of hairy cell leukemia (8), in $60 \%$ of thyroid carcinomas, in $10 \%$ of colorectal carcinomas, and in a minor percentage of nonsmall-cell lung carcinomas (NSCLCs) (9). However, no previous study has reported BRAF V600E mutations in thymic carcinomas.

To our knowledge, this is the first reported case of thymic adenocarcinoma with the nuclear expression of TTF-1 and a BRAF V600E mutation, mimicking lung adenocarcinoma. We report this as a suggestive case for future treatment planning because it reminds us of the further need for genetic evaluations of thymic carcinomas.

\section{Case Report}

A 50-year-old woman attended regular outpatient clinic visits because of bronchial asthma and ulcerative colitis. The patient was a never-smoker. She presented with persistent suffocation and chest pain. Chest high-resolution computed tomography (HRCT) revealed a solid tissue of $42 \times 28 \mathrm{~mm}$ with a smooth surface in the anterior mediastinum (Fig. 1A). ${ }^{18}$ F-Fluorodeoxyglucose positron-emission tomography revealed a high uniform accumulation with a maximum standardized uptake value of 13.4 in the mediastinal mass, and no abnormalities were found at any other

\footnotetext{
${ }^{1}$ Department of Respiratory Medicine, Sapporo Kosei General Hospital, Japan, ${ }^{2}$ Department of Surgical Pathology, Sapporo Kosei General Hospital, Japan, ${ }^{3}$ Department of Surgery, Sapporo Kosei General Hospital, Japan and ${ }^{4}$ Department of Respiratory Medicine and Allergology, Sapporo Medical University School of Medicine, Japan

Received: March 24, 2021; Accepted: June 6, 2021; Advance Publication by J-STAGE: July 17, 2021

Correspondence to Dr. Takafumi Yorozuya, taka0703@sapmed.ac.jp
} 


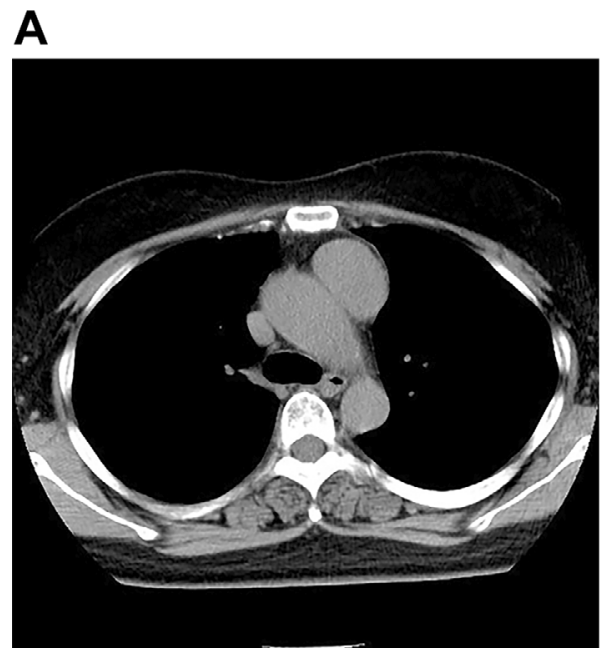

\section{B}

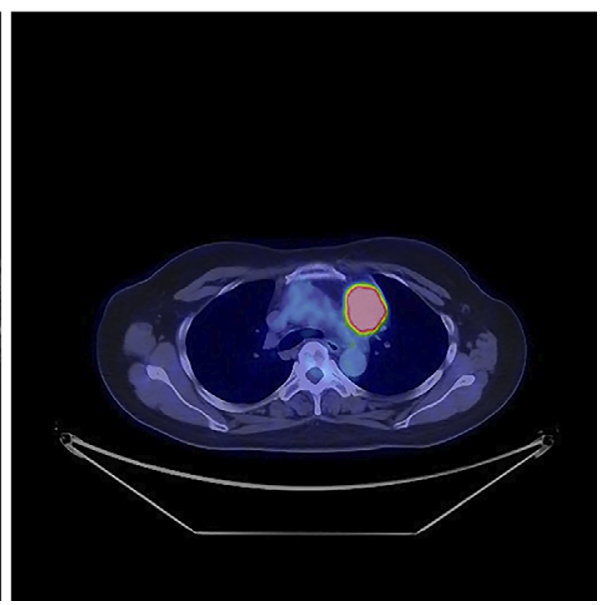

Figure 1. Imaging findings before the surgery. Chest high-resolution computed tomography (HRCT) shows a tumor of $42 \times 28-\mathbf{m m}$ in size with a smooth surface in the anterior mediastinum (A). 18F-fluorodeoxyglucose positron-emission tomography shows a high uptake with a maximal standardized uptake value of 13.4 (B). Chest HRCT shows that the tumor has grown to $46 \times 32 \mathrm{~mm}$ just before the surgery $(\mathrm{C})$.

sites, including both lungs and mediastinal lymph nodes (Fig. 1B). Other examinations revealed no other findings, such as tumors in other parts of the body, including the stomach, colon, uterus, and ovary.

The serum carcinoembryonic antigen (CEA) level was elevated to $37.6 \mathrm{ng} / \mathrm{mL}$. Three months after the onset of symptoms before the surgery, chest HRCT revealed that the tumor had increased in size to $46 \times 32 \mathrm{~mm}$, and the serum CEA level had increased to $55.2 \mathrm{ng} / \mathrm{mL}$. The patient underwent tumor resection using video-assisted thoracoscopic surgery.

Macroscopically, the tumor was located inside the thymus and showed no invasion outside the thymic adipose tissue. The tumor had no apparent blood supply via extracervical vessels. Sectioning demonstrated that the solid lesion was totally encapsulated, well demarcated, and $60 \mathrm{~mm}$ in diameter (Fig. 2A). Focal necrosis and spotty necrosis were observed. No cystic lesions or ectopic thyroid tissue were found. Histologically, the lesion consisted of an adenocarcinoma component with a background thymic lymphoid stroma (Fig. 2B) that contained T-cells with sarcoid reaction and positive staining for CD5 and TdT (Fig. 2C). The cancer cells consisted of complex tubules and tubulopapillary structures lined by columnar cells. Nuclear atypia was moderately to markedly severe, and distinct nucleoli were found. Carcinoma showed no nuclear features of a papillary thyroid carcinoma, such as intranuclear cytoplasmic pseudoinclusions or nuclear grooves. Immunohistochemically, the adenocarcinoma showed positive staining for cytokeratin AE1/ AE3, CEA, CK7, and TTF-1 (Fig. 2D); focal staining for CK19; and negative staining for CD5 (Fig. 2E), TdT, c-KIT, CD30, AFP, glypican 3, hCG, CK20, and thyroglobulin (Fig. 2F). Based on these pathological findings, primary thymic adenocarcinoma, not otherwise specified, was diag- nosed.

An outsourced genetic analysis (by Oncomine Dx Target Test, SRL, Japan) revealed a BRAF V600E mutation. Postoperative radiotherapy was started 2 months after the surgery in accordance with the guideline (10-12), with a 55-Gy radiation dose. The patient developed mild radiation pneumonia that required no treatment. Seven months after the surgery, no apparent imaging findings suggested relapse. The serum CEA level decreased back to within the normal range. The patient provided her oral informed consent for the publication of this report.

\section{Discussion}

The most common histological type of thymic carcinoma is squamous cell carcinoma. Thymic adenocarcinoma is rare, with an incidence of only $3-4 \%$ among thymic carcinomas $(5,6)$. TTF-1 is specifically expressed in pulmonary adenocarcinoma, small-cell lung carcinoma, and papillary thyroid carcinoma. Matoso et al. reported that the TTF-1 positivity rate in other carcinomas was low, being $5.1 \%$ for bladder cancer, $2.5 \%$ for colon cancer, and $1.2 \%$ for prostate cancer (13). Comforti et al. reported that all 29 cases of thymic carcinoma were negative for TTF-1 (14). Only one case of thymic adenocarcinoma positive for TTF-1 has been reported in the literature (7).

In the present case, the tumor clearly originated from the thymus, given the presence of thymus-derived CD5+/TdT+ lymphocytes in the background stroma of the tumor. However, owing to the positivity for TTF-1 expression, making inferences regarding the primary site of the tumor, its growth pattern, and development history is difficult. This is due to the absence of features of conventional thymoma components or thymic cysts and the inability to find the tra- 
A

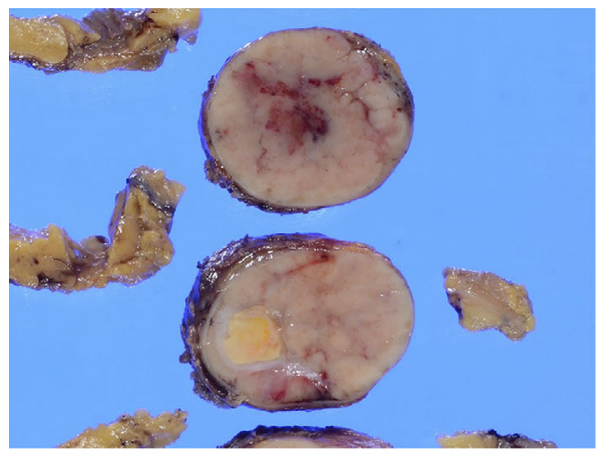

\section{C}

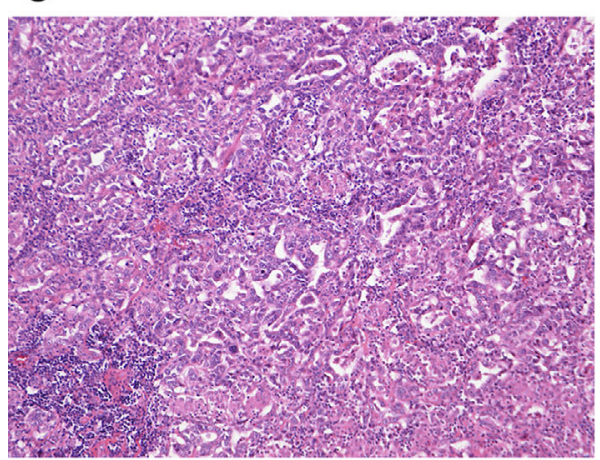

E

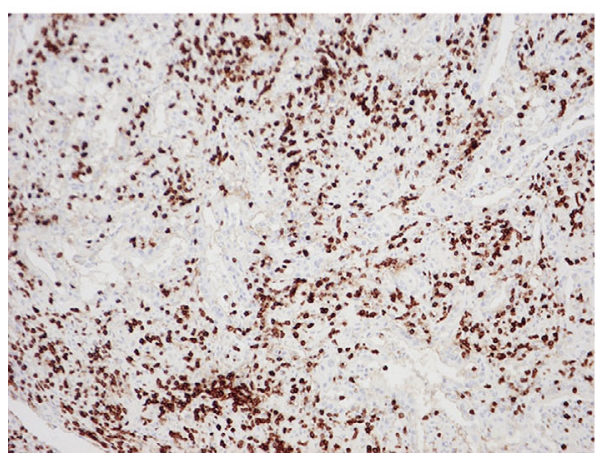

B

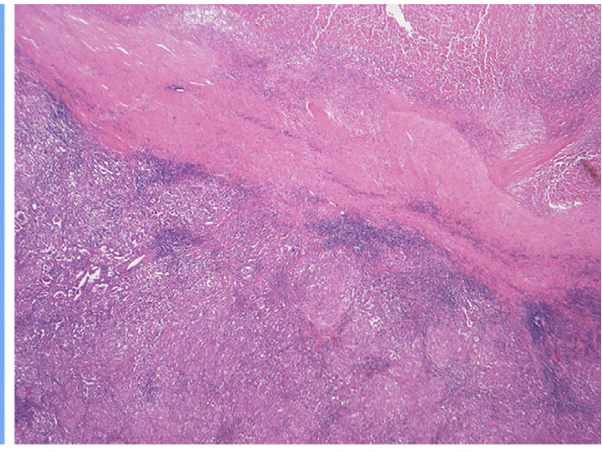

D

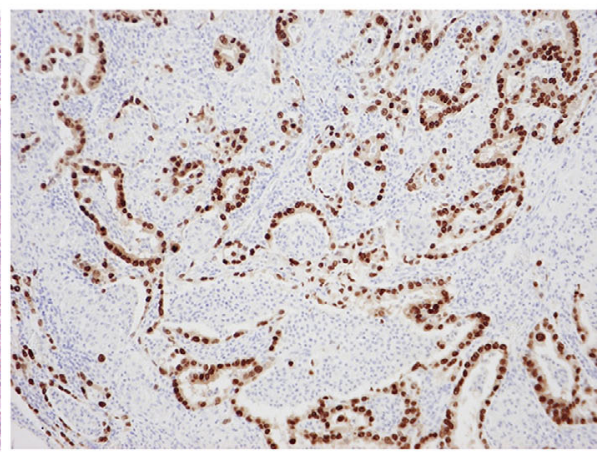

$\mathbf{F}$

Figure 2. (A) Macroscopic appearance of the anterior mediastinal mass measuring $60 \mathrm{~mm}$ in diameter. (B) Hematoxylin and Eosin (H\&E) staining, in the low-power field. The lesion consisted of an adenocarcinoma component with a background thymic lymphoid stroma, sarcoid reaction, and focal necrosis. (C) H\&E staining in the high-power field. The cancer cells consist of complex tubules and tubulopapillary structures lined by columnar cells. Moderately to markedly severe nuclear atypia and distinct nucleoli can be observed. (D) Immunohistochemical staining of adenocarcinoma cells positive for thyroid transcription factor 1 (TTF-1). (E) Only the background lymphoid cells show positive staining for CD5. (F) Cancer cells negative for thyroglobulin.

ditional zone from the thymic in situ component to the adenocarcinoma. The important differential diagnoses are metastasis of thyroid cancers and primary carcinoma arising from ectopic thyroid tissue in the anterior mediastinum. In our case, the cancer cells lacked the nuclear characteristics of a primary thyroid papillary carcinoma and showed negative staining for thyroglobulin. In addition, CT revealed no blood supply via the extracervical vessels as observed in ectopic thyroid tissue. Furthermore, metastasis of lung cancer is difficult to distinguish from primary thymic carcinoma with TTF-1 expression. We found no evidence of malignancy in the lung parenchyma in the present case; however, there are cases wherein metastases may be detected first, and the primary tumor may become evident several years later, although this is rare. One such case was that of primary lung cancer detected five years after surgery for a mediastinal lymph node lesion (15). In addition, metastasis to the thymus is a rare phenomenon. Only one case of colon cancer metastasis to the thymus has been reported (16). Kitamura et al. mentioned that TTF-1 is not always useful for differentiating a primary thymic adenocarcinoma from a metastatic adenocarcinoma originating in the lungs or thyroid, and a comprehensive diagnosis of thymic adenocarcinoma based on the clinical course and histological and radiologi- 
cal findings is important (7). The observation period in this study was short; therefore, although we consider the present case to be thymic in origin at this time, a different primary site may be revealed during long-term follow-up.

BRAF V600E is a mutation present in almost all cases of hairy cell leukemia (8), in $59 \%$ of thyroid cancers, in $51 \%$ of melanomas, in $10 \%$ of colorectal carcinomas, and in $6.7 \%$ of NSCLCs (9). However, no previous studies have reported BRAF V600E mutations in thymic carcinoma or thymoma. According to Sakane et al. none of the 54 cases of thymic carcinoma had a BRAF V600E mutation (17). Our case may be the first reported case of BRAF V600E-mutant thymic carcinoma.

The present patient underwent radiotherapy after complete resection of Masaoka stage II thymic carcinoma. In case of relapse, the treatment policy was discussed. Carboplatin + paclitaxel or carboplatin + amrubicin are treatment options for relapsed thymic carcinoma (18-20). However, the standard treatment for patients with thymic carcinoma previously treated with chemotherapy has not been established. Recently, phase II studies for nivolumab, pembrolizumab, pemetrexed, and S-1 have been conducted (21-24). The characteristics of the cancer cells in the present case may have been the same as those of lung adenocarcinoma, and the guidelines for drug treatment of lung adenocarcinoma may also be applicable (25). Because the patient was BRAF V600E mutant-positive, BRAF inhibitors are a treatment option. However, the response to BRAF inhibitors differed from the response to the type of carcinoma (26). Determination of the clinical significance of positivity for TTF-1 and BRAF mutations in thymic adenocarcinomas will further our understanding of their behavior and may aid in their detection and treatment. More cases are expected to be accumulated, and further clinical trials will be conducted.

The authors state that they have no Conflict of Interest (COI).

\section{Acknowledgement}

The authors would like to thank Enago (http://www.enago.jp) for the English language review.

\section{References}

1. Travis WD, Brambilla E, Burke AP, et al. World Health Organization Classification of Tumors of the Lung, Pleura, Thymus and Heart. IARC Press, Lyon, 2015.

2. de Jong WK, Blaauwgeers JL, Schaapveld M, Timens W, Klinkenberg TJ, Groen HJ. Thymic epithelial tumours: A population-based study of the incidence, diagnostic procedures and therapy. Eur J Cancer 44: 123-130, 2008.

3. Ruffini E, Detterbeck F, Raemdonck DV, et al. Tumours of the thymus: a cohort study of prognostic factors from the European Society of Thoracic Surgeons database. Eur J Cardiothorac Surg 46: 361-368, 2014.

4. Girard N, Ruffini E, Marx A, Faivre-Finn C, Peters S; ESMO Guidelines Committee. Thymic epithelial tumours: ESMO Clinical Practice Guidelines for diagnosis, treatment and follow-up. Ann Oncol 26: v40-55, 2015.

5. Kalhor N, Moran CA. Primary thymic adenocarcinomas: a clinico- pathological and immunohistochemical study of 16 cases with emphasis on the morphological spectrum differentiation. Hum Pathol 74: 73-82, 2018.

6. Koizumi T, Otsuki K, Tanaka Y, et al. National incidence and initial therapy for thymic carcinoma in Japan: based on analysis of hospital-based cancer registry data, 2009-2015. Jpn J Clin Oncol 50: 434-439, 2020.

7. Kitamura M, Okada H, Miyahara S, et al. Case of primary thymic adenocarcinoma associated with type $\mathrm{AB}$ thymoma and thymic cysts. Pathol Int 70: 370-372, 2020.

8. Tiacci E, Trifonov V, Schiavoni G, et al. BRAF mutations in hairy-cell leukemia. N Engl J Med 364: 2305-2315, 2011.

9. Holderfield M, Deuker MM, McCormick F, McMahon M. Targeting RAF kinases for cancer therapy: BRAF-mutated melanoma and beyond. Nat Rev Cancer 14: 455-467, 2014.

10. Jackson MW, Palma DA, Camidge DR, et al. The impact of postoperative radiotherapy for thymoma and thymic carcinoma. J Thorac Oncol 12: 734-744, 2017.

11. Hamaji M, Shah RM, Ali SO, Bettenhausen A, Lee HS, Burt BM. A meta-analysis of postoperative radiotherapy for thymic carcinoma. Ann Thorac Surg 103: 1668-1675, 2017.

12. Lim YJ, Song C, Kim JS. Improved survival with postoperative radiotherapy in thymic carcinoma: A propensity-matched analysis of surveillance, epidemiology, and end results (SEER) database. Lung Cancer 108: 161-167, 2017.

13. Matoso A, Singh K, Jacob R, et al. Comparison of thyroid transcription factor- 1 expression by two monoclonal antibodies in pulmonary and non-pulmonary primary tumors. Appl Immunohistochem Mol Morphol 18: 142-149, 2010.

14. Conforti F, Tarantino P, Trillo P, et al. Pathological and clinical features of enteric adenocarcinoma of the thymus. A pooled analysis of cases from a reference center and systematic review of the literature. Cancer Treat Rev 92: 102133, 2021.

15. Kawasaki H, Arakaki K, Taira N, et al. Lung cancer detected 5 years after resection of cancer of unknown primary in a mediastinal lymph node: A case report and review of relevant cases from the literature. Ann Thorac Cardiovasc Surg 22: 116-121, 2016.

16. Lee M, Choi SJ, Yoon YH, Kim JT, Baek WK, Kim YS. Metastatic thymic adenocarcinoma from colorectal cancer. Korean $\mathrm{J}$ Thorac Cardiovasc Surg 48: 447-451, 2015.

17. Sakane T, Murase T, Okuda $\mathrm{K}$, et al. A mutation analysis of the EGFR pathway genes, RAS, EGFR, PIK3CA, AKT1 and BRAF, and TP53 gene in thymic carcinoma and thymoma type A/B3. Histopathology 75: 755-766, 2019.

18. Lemma GL, Lee JW, Aisner SC, et al. Phase II study of carboplatin and paclitaxel in advanced thymoma and thymic carcinoma. J Clin Oncol 29: 2060-2065, 2011.

19. Hirai F, Yamanaka T, Taguchi K, et al. A multicenter phase II study of carboplatin and paclitaxel for advanced thymic carcinoma: WJOG4207L. Ann Oncol 26: 363-368, 2015.

20. Inoue A, Sugawara S, Harada M, et al. Phase II study of amrubicin combined with carboplatin for thymic carcinoma and invasive thymoma: North Japan Lung Cancer group study 0803. J Thorac Oncol 9: 1805-1809, 2014.

21. Katsuya Y, Horinouchi H, Seto T, et al. Single-arm, multicentre, phase II trial of nivolumab for unresectable or recurrent thymic carcinoma: PRIMER study. Eur J Cancer 113: 78-86, 2019.

22. Giaccone G, Kim C, Thompson J, et al. Pembrolizumab in patients with thymic carcinoma: a single-arm, single-centre, phase 2 study. Lancet Oncol 19: 347-355, 2018.

23. Gbolahan OB, Porter RF, Salter JT, et al. A phase II study of pemetrexed in patients with recurrent thymoma and thymic carcinoma. J Thorac Oncol 13: 1940-1948, 2018.

24. Tsukita Y, Inoue A, Sugawara S, et al. Phase II study of S-1 in patients with previously-treated invasive thymoma and thymic carcinoma: North Japan lung cancer study group trial 1203. Lung Can- 
cer 139: 89-93, 2020.

25. Varadhachary GR, Raber MN. Cancer of unknown primary site. N Engl J Med 371: 757-765, 2014.

26. Prahallad A, Sun C, Huang S, et al. Unresponsiveness of colon cancer to BRAF (V600E) inhibition through feedback activation of EGFR. Nature 483: 100-103, 2012.
The Internal Medicine is an Open Access journal distributed under the Creative Commons Attribution-NonCommercial-NoDerivatives 4.0 International License. To view the details of this license, please visit (https://creativecommons.org/licenses/ by-nc-nd/4.0/).

(C) The Japanese Society of Internal Medicine Intern Med Advance Publication 